\title{
Bacteriolysis by Zinc Ions-Induced PGN Autolysins, Virucide by ZAP, ZBD, ZnONPs, and Prevaling Zinc Ions-Mediated HCoVs Matter
}

\author{
Dr. Sci. Tsuneo Ishida
}

\author{
2-3-6, Saido, Midori-Ku, Saitama-Shi, Saitama-Ken, $\bar{T} 336-0907$, Japan
}

\begin{abstract}
Bacteriolysis by $\mathrm{Zn}^{2+}$-induced peptidoglycan autolysins, virucide by zinc-finger antiviral protein (ZAP), zinc binding domain (ZBD), zinc oxide nanoparticles (ZnONPs), and current $\mathrm{Zn}^{2+}$ ions-mediated human coronavirus matter are respectively discussed. Bacterial peptidoglycan (PGN) autolysin AmiA for S. aureus amidase is acted on PGN binding and cleavage. The AmiA distinguishes PGN mostly by the peptide, and the cleavage is facilitated by a zinc-activated water molecule, in order to develop new therapeutics against MRSA. Lytic amidase autolysin LytA associates with the cell wall via its zinc-binding motif. The LytB PGN hydrolase responsible for physical separation of daughter cells cleaves the GlcNAc- $\beta$-(1,4)-MurNAc glycosidic bond of PGN building units. LytC, LytD, and LytF are expressed in the same subpopulation of cells and complete flagellar synthesis. Autolysin LytF is endopeptidase in B. subtilis that plays a role in cell separation and hydrolase of the peptide. Endopeptidase of autolysin LytF in B. subtilis plays a role in cell separation and hydrolase of the peptide. Endopeptidase of autolysin LytM is a glycyl-glycyl endopeptidase, hydrolyzing the pentaglycine interpeptide crossbridge. Thus, $\mathrm{Zn}^{2+}$ ions induced PGN autolysins for S. aureus is amidase LytA and endopeptidase LytM that are anticipated to be used as antibacterial potential of endogenous PGN-degrading enzymes against $S$. aureus.
\end{abstract}

Zinc-dependent endopeptidases (Eps) are predicted to hydrolyze PGN to facilitate cell growth that zinc avaliability affects strong activity of cell wall hydrolases, and zur-regulated endopeptidases are present in divergent Gram-negative bacteria. AmiB catalyzes the degradation of PGN in Gram-negative bacteria, resulting in a marked increases of sensitivity to oxidative stress and organic acids. AmiC controls cell separation and PGN fragments release. Eps are predicted to hydrolyze PGN to facilitate cell growth that zinc avaliability affects strong activity of cell wall hydrolases. Eps at outer membrane lipoprotein and amidase, peptidase, and carboxypept idase in PGN layer against E. coli are anticipated to be employed as E. coli cell wall-hydrolyzing enzymes of anti-bacterial potential. Zinc-dependent PGN autolysin of amidases are enhanced the anti-bacterial activities against both Gram-positive and Gram-negative bacteria. The autolysin-mediated bacteriolysis induced bacterial cell death can contribute to the bactericidal activities.

On the other hand enveloped viruses enter cells and initiate disease-causing cycles of replication in virus-cell interaction. The novel $E B V$-induced zinc finger gene $\left(Z N F^{E B}\right)$ including its intronless locus and human protein variants, controls virus entry and exit from cell cycling in activated lymphocytes. Zinc finger protein $(Z N F)$ is prepared consiting HIV-1 type integrase fused to the synthetic zinc finger protein E2C that offers an efficient approach and a versatile framework for directing the integration of retroviral DNA into a predetermined DNA site. ZNF Tsipl controls Cucumber mosaic virus (CMV) RNA replication. ZNF ZCCHC3 binds RNA and facilitates viral RNA that is critical for RIG-1 like receptor (RLR)-mediated innate immune response to RNA virus.

ZAP inhibits entry, replication, and spread of certain viruses, and promotes viral RNA degradation. ZAP may regulate $D N A$ and RNA virus replication that ZAP controls Retroviral RNA production and HIV-1 infection by promoting the degradation of specific viral mRNAs. ZAP-70 kinase regulates HIV cell-to-cell spread that HIV usurps components of the immunnological synapse machinery to ensure its own spread through cell-to-cell contacts.

$Z B D$ is essential for formation of the functional Junin virus envelope glycoprot ein complex. ZBD inhibits Nidovirus $R N A$ synthesis and replication, hence the 2019-nCoV may be regulated by ZBD.

ZnONPs recently are used in various applications of veterinary science due to their antibacterial and antiviral agents, tissue repair that the ZnONPs are anticipated to be employed in prevention of human coronavirus infection.

Today, seventh known HCoVs have been identified, namely HCoVs-229E, HCoV-NL63, HCoV-OC43, HCoV-HKU1, severe acute respiratory syndrome coronavirus (SARS-CoV), Middle East respiratory syndrome coronavirus (MERS$\mathrm{CoV})$, and recently new-typed 2019-nCoV, subsequent phylogenetic studies pointed to the bat origin of SARS-CoV based on sequences of SARS-like virus found in bats. This 2019-nCoV ( $\beta-C o V)$ structure has a spike glycoprotein (S) that the coronavirus protein mediates coronavirus entry into host cells which the evolution of these two critical functions of coronavirus spike proteins, receptor recognition and membrane fusion must be considered to be able to degrade or suppress for the spikes and the membrane by $\mathrm{Zn}^{2+}$-centered t et rahedrally coordinated binding.

Anti-viral activities of $\mathrm{Zn}^{2+}$ ions released from $\mathrm{ZNF}, \mathrm{ZAP}, \mathrm{ZBD}$, and ZnONPs are recognised by which highly diverse fusion proteins have converged on the same overall strategy to mediate a common pathway of membrane fusion, Zinc ion drug development is anticipated to be adopted by using ZAP viral destruction via cell surface receptors and $\mathrm{Zn}^{2+}$ coordination pattern, causing to lead enhancement of the anti-viral activity.

Keywords: Bacterial PGN autolysin, Autolysin amidase, Zinc-finger antiviral protein, Virus entry, replication and spread, Zinc oxide nanoparticles, Human Coronavirus, Prevention of respiratory and pneumonic spreading 
Abbreviations; Aas = auto lysin/ adhesin of Staphylococcus saprophyticus, $\boldsymbol{A} \boldsymbol{B C}=\mathrm{ATP}-\mathrm{binding}$ cassette, $\mathbf{A P C}=$ antigen presenting cell, $\boldsymbol{A}$. stephensi= Anopheles stephensi, B. abortus = Brucella abortus, B. subtilis=Bacillus subtilis, $\quad \mathbf{C B D s}=$ cell wall binding domains, $\mathbf{C B P s}=$ choline binding proteins, C. difficile $=$ Clostridium difficile, $\mathbf{C K D}=$ Chronic Kidney Disease, $\mathbf{C M V}=$ Cucumber mosaic virus, $\boldsymbol{E}$. coli=Escherichia coli, $\boldsymbol{E}$. faecalis = Enterococcus faecalis, ETEC=Enterotoxigenic E.coli, Eps=Zinc dependent endopeptidases, $\boldsymbol{F n B P}=$ fibronectin-binding proteins, Gas=group A streptococcus, GelE= gelatinase, $\mathrm{HCoV}=$ human coronavirus, $\mathrm{HCV}=$ hepatitis C virus, HD=hemodialysis, HIV-1=Human immunodeficiency virus type 1, IFITMs=Interferon transmembrane proteins, $\boldsymbol{M}$. catarrhalis=Moraxella catarrhalis, $\mathbf{M C P s}=\mathbf{M}$ et allocarboxypeptidases, MIBRs $=$ most probable immunoprotective B-cell epitope regions, $\mathbf{M R B}=$ multidrug bacteria, $\mathbf{O R S s}=$ oral rehydration solutions, ORT=oral rehydration therapy, $\boldsymbol{P}$. aeruginosa $=$ Pseudomonas aeruginosa, $\mathbf{P B P 2} \mathbf{a}=$ penicilline-binding protein2a, $\mathbf{P G N = p e p t i d o g l y c a n ,}$ PGRPs=peptidoglycan recognition proteins, PSP= plasmid stabilization protein, $\mathbf{R d R} \mathbf{p}=\mathbf{R N A}$-dependent RNA polymerase, RIG-1=retinoic acid-inducible gene-1, RLR=RIG-1 like receptor, $\mathbf{R O S}=$ reactive oxygen species, $\mathbf{S a g s}=$ super-antigens, $\mathbf{S a s} \mathbf{G}=S$. aureus surface protein, $\boldsymbol{S}$. aureus=Staphylococcus aureus, $\mathbf{S B P}=$ solute-binding protein, $\mathbf{S E B}=$ staphylococcal entoxin serotype B, SINV=Sindbis virus, $\mathbf{S O D}=$ superoxide dismutase, $\mathbf{S}$. pneumoniae $=$ Streptococcus pneumoniae, $\mathbf{S S P}=$ stable signal peptide, $\mathbf{T B V s}=$ transmission-blocking vaccines, Tsip1=Tsi1-interacting protein 1, VRE=vancomycin-resistant Enterococcus faecium, $\mathbf{Z A P}=$ zinc-finger antviral protein, $\mathbf{Z B D}=$ zincbinding domain, $\mathbf{Z B L}=$ zinc binding lipoprotein, $\mathbf{Z N F}^{\mathbf{E B}}=\mathrm{EBV}$-induced zinc finger gene, $\mathbf{Z n O N P s = z i n c}$ oxide nanoparticles, ZnuA=Zinc uptake A.

\section{INTRODUCTION}

Zinc is a nutritionally fundamental trace element in human body that the recommended daily intake of zinc depends on several factors. Average values of recommended intake may be $711 \mathrm{mg} /$ day for adults. Zinc deficiency currently accounts for approximately $16 \%$ of lower respiratory tract infections, $18 \%$ of malaria, and $10 \%$ of diarrheal diseases, while severe zinc deficiency is rare, mild to moderate deficiency is more common worldwide [1]. Zinc homeostasis is a key factor in maintaining a healthy immune system that zinc homeostasis during acute phase response is the temporal transfer of serum zinc to the tissues, causing transient serum hypozincemia, which is rebalanced during resolution of the inflammatory response that intracellularly increased zinc can intoxicate engulfed pathogens and acts cytoprotective by promotion of neutralizing reactive oxygen species (ROS) and reactive nitrogen species (RNS) [1]. The other, zinc excess provokes an impairment of the immune system and has significant toxicity to bacteria.

Zinc is known to be essential for highly growth and development of all organisms in the human body, especially the immune system. A variety of effects of zinc on immune cells depend on the zinc concentration that in a concentration of 100 $\mu \mathrm{mol} / \mathrm{L}$, zinc suppresses natural killer cell killing and T-cell function whereas monocytes are activated direcly, and in a concentration of $500 \mu \mathrm{mol} / \mathrm{L}$, zinc evokes a direct chemotactic activation of neutrophil granulocytes [2]. The zinc ions play important roles of effects on prevention and reduction for bacterial and viral infections. Bacteria and viruses are most common cause of foodborne disease by food poisoning and many human illnesses are caused by bacterial and viral infections. Bacterial and viral infections are associated with deficiencies in macronutrients and micronutrients, including the essential trace elements that dietary supplementation to provide adequate element supply has been proposed to confer health benefits for patients suffering from some bacterial or viral diseases [3]. The treatments with herbal nutraceuticals and zinc likely indirectly contributed to the increase in the resistance of the lambs against Haemonchus contortus infection [4]. Zinc is the second most abundant trace metal with human body $2-3 \mathrm{~g}$ and a plasma concentration of $12-16 \mu \mathrm{M}, 90 \%$ in muscle and bone, and $10 \%$ other organs include prostate, liver, the gastrointestinal tract, kidney, skin, lung brain, heart, and pancreas in humans that cellular zinc underlies an efficient homeostatic control that avoids accumulation of zinc in excess. Host zinc homeostasis changes in response to bacterial infections, including production of metal sequestering proteins and bombardment of bacteria with toxic level of zinc at host-pathogen interface [5].

Apoptosis is defined as cell death activated by an internally controlled suicide program that bacteria are able to trigger apoptosis, including the secretion of compounds such as protein synthesis inhibitions, pore forming proteins, molecules responsible for the activation of the endogeneous death in the infected cell, and super antigens [6]. Zinc influences apoptosis by acting on several molecular regulators of programmed cell death and zinc deficiency caused by malnutrition and foods with low bio- 
availability, aging, certain diseases, and deregulated homeostasis is a far more common risk to human health without intoxication [7]. The influence of zinc on apoptosis is tissue/cell type, zinc concentration, and expression of zinc transporters and zinc-binding proteins.

The other, zinc deficiency in Chronic Kidney Disease (CKD) patients may be due to fecal excretion or decrease in its absorption that zinc concentrations were lower in hemodialysis (HD) patients compared to controls and $\mathrm{Zn}$ concentration $69.16 \mu \mathrm{g} / \mathrm{dL}$ of blood in HD patients, however, revealed no correlation among serum $\mathrm{Zn}$ concentration and anemia, serum parathyroid hormone concentration or pruritus severity in HD patients [8].

Bacterial killing of $\mathrm{Zn}^{2+}$ ions occurs chiefly by bacteriolyses of bacterial cell walls due to activated peptidoglycan (PGN) autolysins such as amidases, endopeptidases, and carboxypeptidase against bacteria [9]. PGN autolysins induced anti-bacterial vaccine activity may be enhanced by activation of zinc dependent PGN autolysins. PGN autolysins are bacterial peptidoglycan degrading enzymes that these muropeptides can be produced or modified by the activity of bacterial glycolytic and peptidolytic enzymes referred to as PGN hydrolases and autolysins which specific bacterial pathogens use PGN degradation to subvert host innate immunity [10]. Bact-eria have to avoid recognition by the host immune system in order to establish a successful infection which bacterial autolysins enable the bacteriolyses of bacterial cell walls trim cell surface PGN to prevent detection by bacterial innate immune system [11].

Viruses are intracellular obligate parasites that cause infection by invading cells of the body. Their life cycle comprises a short extracellular period and a longer intracellular period during which they undergo replication. The immune system has non-specific and specific mechanism that attacks the virus in both phases of its life cycle which specific antibodies protect against viral infections and play an important role in antiviral immunity, mainly during the early stage of the infection [12]. To date, there are presence as severe problems in the world against the infection of new-typed human coronavirus (HCoV) of 2019-novelCoV. The rapid final completion is anticipated.

In this review, firstly, bacteriolysis of bacterial cell walls by $\mathrm{Zn}^{2+}$ ions induced autolytic PGN activation are debated against St aphylococcus aureus (S. aureus) as Gram-positive bacterium and Escherichia coli (E. coli) as Gram-negative bacterium. Secondly, the zinc-mediated antiviral immunity and viral inhibition by zinc-finger protein, zinc-finger antiviral protein, zincbinding domain, and zinc oxide nanoparticles are discussed, and $\mathrm{Zn}^{2+}$ ions-mediated antivirus against Human Coronaviruses also is dealt with. Lastly, the bacteriolytic and the virucidal mechanisms on the bacterolysis by $\mathrm{Zn}^{2+}$ ionsinduced PGN autolytic activation and on the ZAP-induced virucide strain are respectively clarified.

ZINC-INDUCED BACTERIAL AND VIRAL IMMUNITY TO MAINTAINE CONSTANT HomeOSTASIS

Zinc-induced anti-bacterial immunity is important factor to be both essential and toxic for microorganism that zinc (- ${ }_{-}^{+}$i)ions have crucial roles in many facets of the immune system, in which microbial susceptibility to $\mathrm{Zn}$ (II) toxicity is mediated by extracellular cation competition and this susceptibility can be harnessed by innate immune response [13].

It is essential for bacteria to maintain metal ion homeostasis that the need for tight homeostatic control is particularly true for zinc, an essential transition metal ion which zinc ions may be used as an antimicrobial agent in the innate immune system and zinc efflux is an important contributor to group A Streptococcus (GAS) pathogenesis [14]. Maintaining a constant state of cellular zinc homeostasis is essential for normal function that typically, human zinc intakes range from 107 to $231 \mu \mathrm{mol} / \mathrm{dm}^{3}$; this is equivalent to $14 \sim 30 \mathrm{mg} / \mathrm{kg}$ for comparison with rat diets [15]. Zinc-modulating immune response depends on a sufficient availability of this zinc that zinc supplementation in diseases diarrhera, chronic hepatitis pneumonia, and acute lower respiratory infection seems beneficial [16]. Zinc induced antiviral immunity plays an important role in antiviral antibodies that zinc binds to the viral envelope or capsid proteins, and block the virus from entering into host cell, in which cytotoxic $\mathrm{T}$ lymphocytes (CTL) cells in specific antiviral immunity recognize viral antigens presented at the cell surface associated with I major histocompatibility complex (MHC) molecule [17]. An essential trace element zinc is crucial for growth, development, and the maintenance of immune function which zinc status is a critical factor that can influence antiviral immunity, particularly as zinc-deficient 
populations are often most at risk of acquiring viral infections such as HIV, HCV [18]. Common features possess that enveloped viruses enter cells by membrane-fusion protein on the surface, fusion glycoprotein on metastable prefusion and interactions with neutralizing antibodies. Implications for immunogen design of next-generation vaccines have been shown from the results that stable immunogens presenting the same antigenetic sites as the labile wild-type proteins efficiently elicit potently neutralizing antibodies [19].

BACTERIOLYSES BY $\mathrm{ZN}^{2+}$ ION-INDUCED PGN AUTOLYSINS

Molecular Structures of S. Aureus and E. coli Cell Walls and the Action Sites of PGN Autolysins

Bacterial PGN structure of both Gram-positive and Gram-negative bacteria comprises repeating disaccharide backbones of $\mathrm{N}$-acetylglucosamine (NAG) and $\beta-(1-4)-\mathrm{N}$-acetylmuramic acid (NAM) that are crosslinked by peptide stem chains attached to the NAM residues [20]. The action sites of bacterial autolysins are comprised that for Staphylococcus aureus (S. aureus) PGN layer cell wall, there are $\mathrm{N}$-acetylmuramidase-Lalanine amidase and PGN chain cross-linkage DD-endopeptidase.

The other, for Escherichia coli (E. coli) cell wall, there are endopeptidase of degrading enzyme at lipoprotein of $\mathrm{C}$ - and $\mathrm{N}$-terminals, and amidase, peptidase, and caboxypeptidase at thin PGN layer in periplasmic space [21]. The bacterial cell walls are a strong flexible mesh work of PGN that gives a bacterium structural integrity, in which to accommodate a growing cell, the walls are remodeled by PGN synthesis and PGN autolysin. PGN is the main constituent of bacterial cell walls and must be continuously synthesized and degraded to maintain the integrity and viability of the cells that bacterial cell wall hydrodases of amidase,gycosidase, and peptidase display a modular architecture combining multiple and different catalytic domains, including some lytic transglycosylases as well as cell wall binding domains [22]. In these autolysins, zinc-dependent PGN autolysin of amidases may be enhanced and induced anti-bacterial activities.

\section{$\mathrm{Zn}^{2+}$ Ions Induced Activated PGN Autolysins Promote the Bacteriolysis against Gram- Positive Bacterial Cell Wall}

S.aureus amidase AmiA is acted on PGN binding and cleavage. The AmiA distinguishes
PGN mostly by the peptide, and the cleavage is facilitated by a zinc-activated water molecule, in order to develop new therapeutics against MRSA [23]. The autolytic activity of the recombinant amidase of the Aas (autolysin/adhesin of Staphylococcus saprophyticus) is inhibited and is neccesary for the C-terminal GW repeats, not the $\mathrm{N}$-terminal repeats [24].

Lytic amidase autolysin LytA which is released by bacterial lysis, associates with the cell wall via its zinc-binding motif that the amidase domain comprises a complex substrate-binding crevice and needs to interact with a large-motif epitope of PGN for catalysis [25]. Suicidal amidase autolysin LytA having both autolysis and capsule shedding depends on the cell wall hydrolytic activity of LytA that capsule shedding drastically increases invasion of epithelial cells and is the main pathway by which pneumococci reduce surface bound capsule during early acute lung infection of mice [26]. The LytB PGN hydrolase responsible for physical separation of daughter cells cleaves the GlcNAc- $\beta$ - $(1,4)$-MurNAc glycosidic bond of PGN building units that cell wall digestion products and solubilisation rates might indicate a tight control of LytB activity to prevent unrestrained breakdown of the cell wall [27]. The PGN-remodeling autolysins LytC, LytD, and LytF are expressed in the same subpopulation of cells and complete flagellar synthesis that LytC appears to be important for flagellar function, motility was restored to a LytC mutant by mutation oef either lon A, and LytC, LytD, and LytF autolysins to population heterogeneity in B. subtilis [28].

Atl is the major autolysin in $S$ aureus that the bifunctional major autolysin play a key role in staphylococcal cell separation which processing of Atl yield catalytically active amidase and glucosamidase domains [29]. The biochemical and strucural staphylococcal Atl have successful cloaning, high level over-expression, and purification Atl proteins [30]. AtlA is the major PGN hydrolases of Enterrococcus faecalis involved in cell division and cellular autolysis and the zinc metalloprotease, gelatinase (GelE) of their interplay proposed to regulate AtlA function, which N-terminal cleavage was required for efficient AtlA-mediated cell division, and AtlA septum localization and subsequent cell separation can be modulated by a single GelE-mediated N-terminal cleavage event[31]. Major Atl autolysin also have an essential role in the early events of the 
fibronectin-binding proteins (FnBPs)-dependent S.aureus biofilm phenotype [32]. For the contribution of autolysins of PGN hydrolases to bacterial killing, t here are $\mathrm{N}$-acet yl-glucosa minidase (At 1A), two N-acetyl-muraminases (AtlB and AtlC) [33].

Endopeptidase of autolysin LytF in B. subtilis plays a role in cell separation and hydrolase of the peptide [34]. Endopeptidase of autolysin LytM is a glycyl-glycyl endopeptidase, hydrolyzing the pentaglycine interpeptide crossbridge [35].

Thus, $\mathrm{Zn}^{2+}$ ions induced PGN autolysins for $S$. aureus is amidase LytA and endopeptidase LytM that are anticipated to be used as antibacterial potential of endogenous PGNdegrading enzymes against $S$. aureus [36].

$\mathrm{Zn}^{2+}$ Ions Induced Degrading Enzyme of Outer Membrane Lipoprotein and PGN Autolysins Promote the Bacteriolysis against GramNegative Bacterial Cell Wall

Zinc-dependent endopeptidases (Eps) are predicted to hydrolyze PGN to facilitate cell growth that zinc avaliability affects strong activity of cell wall hydrolases, and zurregulated endopeptidases are present in divergent Gram-negative bacteria [37].

Amidase gene (AmiB) catalyzes the degradation of PGN in Gram-negative bacteria that the amiB gene was composed of 1,722 nucleotides and 573 amino acid which is involved in the separation of daughter cells after cell devision and inactivation of the amiB gene, resulting in a marked increases of sensitivity to oxidative stress and organic acids [38]. Amidase activity of amiC controls cell separation and PGN fragments release [39].

Zinc-regulated peptidase maintains cell wall integrity during immune-mediated nutrient sequestration against Acinetobacter baumannii [40].

Table1. Zinc induced bacteriolysis against Gram-positive thick PGN envelope cell wall and Gram-negative lipoprotein and thin PGN layer cell wall

\begin{tabular}{|c|c|}
\hline $\mathrm{Zn}^{2+}$ Ions & Gram-Positive PGN Layer Cell Wall \\
\hline $\mathbf{Z n}^{2+}$ & $\begin{array}{l}\longrightarrow \mathrm{Zn}^{2+}, \mathrm{O}_{2}^{-}, \mathrm{H}_{2} \mathrm{O}_{2}, \cdot \mathrm{OH}, \cdot \mathrm{NO}, \mathrm{ONOO}^{-} \\
\mathrm{Zn}^{2+} \text { ions induced activated PGN autolysins } \\
\text { - S.aureus amidase AmiA } \\
\text { - Recombinant amidase of the Aas } \\
\text { - Lytic amidase LytA for Streptococcus pneumoniae } \\
\text { - Pneumococcal autolysin LytA LytC, D, F of PGN remodeling for Bacillus } \\
\text { subtilis } \\
\text { - Endopeptidase LytF for bacillus subtilis } \\
\text { - AtlA autolysin for GelE against } E \text {. faecalis } \\
\text { - AtlA, AtlB, AtlC autolysins against enterococcus faecalis } \\
\text { - Fusion protein autolysin,MIBRs against } S \text {. pneumoniae } \\
\text { - Metallocarboxypeptidase M32 against Trypanosoma brucei or cruzi } \\
\text { - PBP2a and autolysin mixture aginst MRSA }\end{array}$ \\
\hline
\end{tabular}


Bacteriolysis by Zinc Ions-Induced PGN Autolysins, Virucide by ZAP, ZBD, ZnONPs, and Prevaling Zinc Ions-Mediated HCoVs Matter

\begin{tabular}{|c|c|c|}
\hline $\mathrm{Zn}^{2+}$ ions & \multicolumn{2}{|l|}{ Gram-Negative Cell Wall } \\
\hline & $\begin{array}{l}\text { Outer Membrane Lipoprotein } \\
\text { at } \mathrm{C} \text { - and N-terminals }\end{array}$ & $\begin{array}{l}\text { Periplasmic Space Thin PGN } \\
\text { Layer }\end{array}$ \\
\hline $\mathrm{Zn}^{2+}$ & $\begin{array}{l}\longrightarrow \quad \mathrm{Zn}^{2+}, \mathrm{O}_{2}^{-}, \mathrm{H}_{2} \mathrm{O}_{2} \\
\cdot \text {-Amidase gene amiB/LysM } \\
\cdot \text { - Endopeptidase regulation of ShyA } \\
\text { and ShyB } \\
\cdot \text {-Outer membrane receptor against } \\
\text { N.menigitidis } \\
\text {-ETEC subunit vaccine } \\
\text {-ZnuB against } P \text {. aeruginosa. } \\
\text { - Preventive vaccine by recombinant } \\
\text { flagella against } P \text {. aeruginosa }\end{array}$ & 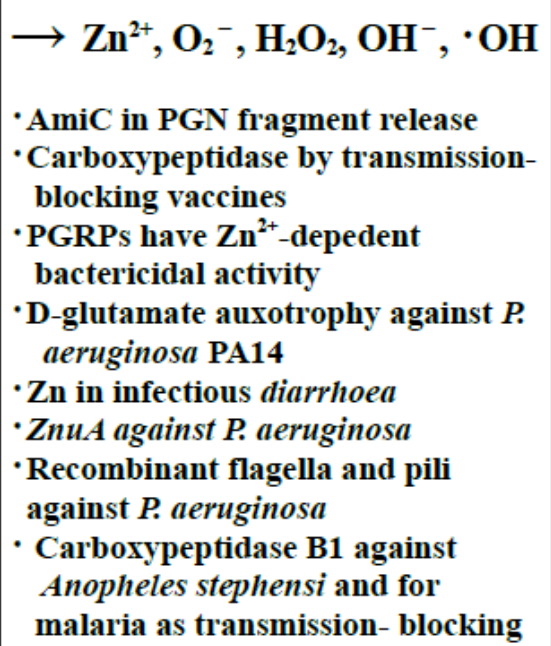 \\
\hline
\end{tabular}

VIRUCIDAL ACTIVITIES OF ZINC-FINGER Antiviral Protein, ZinC-Binding DOMAIN, AND ZINC OXIDE NANOPARTICLES

\section{Zinc-Finger Protein}

Zinc salts $\left(\mathrm{Zn}^{2+}\right.$ concentration: $\left.<80 \mu \mathrm{M}\right)$ suppressing the activity of viral RNA-dependent RNA polymerase, brock hepatitis E virus (HEV) replication [44]. Respiratory syncyt ial vitrus (RSV) is the most important viral cause of acute respiratory tract infection (ARI) that the complete inhibitory effect of zinc salts $\left(\mathrm{Zn}^{2+}\right.$ concentration: 1 and $10 \mathrm{mM}$ ) on RSV plaque formation was observed, in which zinc (_+i) ions mediate antiviral activity on RSV by altering the ability of the cell to support RSV replication [45].

The novel EBV-induced zinc finger gene $\left(Z N F^{E B}\right)$ including its intronless locus and human protein variants, controls entry and exit from cell cycling in activated lymphocytes [46]. The designed polydactyl zinc-finger protein (ZNF) is prepared consiting HIV-1 type integrase fused to the synthetic zinc finger protein $\mathrm{E} 2 \mathrm{C}$ that offers an efficient approach and a versatile framework for directing the integration of retroviral DNA into a predetermined DNA site [47]. The ZNF ZCCHC3 binds RNA and facilitates viral RNA that $\mathrm{ZCCHC} 3$ is a co-receptor for the retinoic acid-inducible gene-1 (RIG-1) and antigen MDA5 which is critical for RIG-1 like receptor (RLR)-mediated innate immune response to RNA virus [48]. Artificial ZFN were targeted to the high affinity Sp1-binding site, and by being fused with TatdMt and POZ domain, they strongly block both Sp1-cyclin T1-dependent transcription and Tat-dependent transcription of HIV-1 [49]. ZNF Tsip1 that the candidate genes encoded Tsi1-interacting protein 1 (Tsip1), a zinc ( $\mathrm{Zn})$ finger protein Tsip1 strongly interacted with CMV $2 \mathrm{a}$ protein, controls Cucumber mosaic virus (CMV) RNA replication [50].

\section{Zinc-Finger Antiviral Protein}

The zinc-finger antiviral protein (ZAP) controls virus entry, DNA/RNA replication, and spreading against viral infection. The ZAP in first steps of $\mathrm{HCV}$ infection may be used as entry inhibitor [51]. Interferon induced transmembrane proteins (IFITMs) inhibit the cellular entry of a broad range of viruses that IFITM-mediated restriction requires recognition of viral RNA elements [52]. The interferonstimulated genes serve as enhancers of antiviral innate immunity [53]. ZAP inhibits alphavirus replication that elucidation of the antiviral mechanism by which ZAP inhibits Sindbis virus (SINV) translation may lead to the development of agents with broad activity against alphaviruses [54]. The ZAP also inhibits Influenza A virus (IAV) protein expression, in which suggests an important role of ZAP in the host effort to control IAV infection and the importance of the threat of ZAP to the virus [55]. The host cell restriction factors that limit IAV have been investigated [56]. ZAP may regulate DNA and RNA virus replication. Inhibition of bacterial DNA replication during 
nitrosative stress is accompanied by zinc mobilization [57]. ZAP specifically inhibits the replication of certain viruses and promotes viral RNA degradation [58]. ZAP inhibits Retroviral RNA production [59] and ZAP inhibits HIV-1 infection by promoting the degradation of specific viral mRNAs [60].

\section{ZAP Regulates Spread}

ZAP' stress with antiviral activity and induced virus replication are regulated upon virus infection to inhibit virus spread [61]. ZAP-70 kinase regulates HIV cell-to-cell spread that HIV usurps components of the immunnological synapse machinery to ensure its own spread through cell-to-cell contacts [62]. An understanding of viral cell-to-cell transmission spreading will enhance our ability to intervene in the efficient spreading of viral infection [63].

\section{Zinc-Binding Domain}

A novel zinc-binding domain (ZBD) is essential for formation of the functional Junin virus envelope glycoprotein complex that the envelope glycoprotein of the Junin arenavirus (GP-C) mediates entry into target cells through a $\mathrm{pH}$-dependent membrane fusion mechanism, in which this unusual motif may act to retain a cleaved 58-amino-acid stable signal peptide (SSP) for its role in modulating membrane fusion activity [64]. Entry of the virus into the host cell is mediated by the viral envelope glycoprotein, GPC that SSP was retained in GPC through interaction with a zinc-binding domain (ZBD) in the cytoplasmic tail of transmembrane fusion of $\mathrm{G} 2$ subunits that Junin virus $\mathrm{ZBD}$ displays a novel fold containing two zinc ions, in which the structural basis for retention of the unique SSP submit suggests a mechanism whereby SSP is positioned in the GPC complex to modulate $\mathrm{pH}$-dependent membrane fusion [65]. In addition, ZBD inhibits Nidovirus RNA synthesis and replication [66], hence the 2019-nCoV may be regulated by ZBD.

\section{Viral Membrane Fusion Protein}

Enveloped viruses enter cells and initiate disease-causing cycles of replication that in all cases virus-cell fusion is executed by one or more viral surface glycoproteins denoted as the fusion protein, in which the structure and mechanisms on viral membrane fusion protein are important problems [67]. The membrane fusion reaction, membrane interaction, conformational changes of specialized virus envelope proteins, and refolding reactions of specific fusion proteins can mediate both viruscell fusion leading to infection and pathological cell-cell fusion, in which they are increasingly viewed as targets for antiviral intervention [67].

\section{Zinc Oxide and Znonps Inhibit Virus Entry, Replication, and Spread}

Zinc oxide tetrapods (ZnOTs) of micronanostructures block HSV-2 attachment, entry into host cell, and stop the spread of the virus among already infected cells [68]. ZnOTs can inhibit HSV-1 growth and spread in corneal tissues, hence, the ZnOTs also could control HCoVs [69].

Zinc has broad-spectrum antiviral activity against HIV,transmissible gastroenteritis virus (TGEV), and SARS-CoV that $2,500 \mathrm{mg} / \mathrm{kg}$ diet $\mathrm{Zn}^{\text {high }}$ showed a down-regulation of interferon (IFN)- $\alpha$, oligoadenylate synthetase (OAS), Zn transporter (ZIP4), as well as the $\mathrm{Zn}$ transporters (ZnT1) and (ZnT5), in which the $\mathrm{Zn}^{\text {high }}$ documented an earlier and higher sysmatic TGEV-specific serum antibody response [70].

Zinc oxide nanoparticles (ZnONPs) recently are used in various applications of veterinary science due to their antibacterial and antiviral agents, tissue repair that the ZnONPs are anticipated to be employed in prevention of human coronavirus infection. Firstly, ZnONPs inhibit H1N1 influenza virus entry into the host cells [71]. ZnONPs-5 $\mu \mathrm{mg} / \mathrm{ml}$ specifically regulated the correlation of microRNAs and their targeted genes [72]. Zinc oxide nanoparticles to dimethylnitrosamine (DMN) treated rats inhibit the production of mRNA of inflammatory cytokines and reduce lipid peroxidation, oxidative stress and fibrosis in the liver [73].

\section{Zinc Induced Human Coronavirus and Prevention of Respiratory Ailment and Pneumonia against $\mathrm{HCoVs}$}

Coronaviruses (CoVs) and arteriviruses are related human and animal pathogens that belong to the Coronaviridae family in the order Nidovirales that CoVs have the largest known RNA genomes. The RNA-dependent RNA polymerase (RdRp) and enzymatic functions for nidoviruses that are characterized by polycistronic plus-stranded RNA genome are required to suppress the consequences of the typically high error rate of viral RdRps that the RdRp behaviour and interactions during RNA synthesis, and subsequent processing must be 
understandable [74]. Zinc ions are essential for the rescue of the enzymatic activities of nidovirus helicases that a complex zinc finger can inhibit possibly virion biogenesis, nidovirus replication and transcription, and dysrupting RNA synthesis [66].

Human coronaviuses $(\mathrm{HCoVs})$ are recognised as coronaviruses (CoVs) associated with multiple respiratory diseases of varying severity, including common cold, pneumonia and bronchilitis that to date, seventh known HCoVs have been identified, namely HCoVs-229E, HCoV-NL63, HCoV-OC43, HCoV-HKU1, severe acute respiratory syndrome coronavirus (SARS-CoV), Middle East respiratory syndrome coronavirus (MERS-CoV), and recently newtyped 2019-nCoV, subsequent phylogenetic studies pointed to the bat origin of SARS-CoV based on sequences of SARS-like virus found in bats [75]. The new developed drugs for the 2019-nCoV has been now reported that Lopinavir/ Ritonavir, Nucleoside analogues, Neuraminidase inhibitors, Remdesivir, peptide (EK1), abidol, RNA synthesis inhibitors (such as TDF, 3TC), anti-inflammatory drugs (such as hormones and other molecules), Chinese traditional medicine, such Shu Feng JieDu Capsules and Lianhuaqingwen Capsule, could be the drug treatment options for 2019-nCoV. However, the efficacy and safety of these drugs for 2019-nCoV still need to be further confirmed by clinical experiments [76]. $\mathrm{HCoVs}$ are a well-known cause of respiratory infections, in which $\mathrm{Zn}^{2+}$ inhibits coronavirus and arterivirus RNA polymelase activity, and zinc ionophores block the virus replication that the combination of $\mathrm{Zn}^{2+}$ and pyrithione at low concentrations $\left(\mathrm{Zn}^{2+}\right.$ concentration $\left.2 \mu \mathrm{M}\right)$ inhibits the replication of SARS-CoV and arterivirus RNA [77]. High zinc ion concentration $(10$ and $100 \mu \mathrm{M})$ to the addition of compounds and $\mathrm{Zn}$ concentration increasing in $\mathrm{Cu} / \mathrm{Zn}$ brasses were found to inhibit the replication of various RNA virus, influenza viruses, respiratory syncytial virus and human coronavirus 229E [78].

Replication of SARS-CoV requires proteolytic processing of the replicase polyprotein by two viral cystein proteases, a chymotrypsin-like protease (3CLpro) and a papain-like protease (PLpro). This PLpro is important for development of antviral drug that would inhibit viral replication and reduce mortality associated with outbreaks of SARS-CoV that a model of PLpro in complex with ubiquitinaldehyde reveals well defined sites within the catalytic cleft that help to account for str ict substrat e-recognit ion motifs [79]. The MERS-CoV PLpro blocking loop 2 (BL2) structure differs from that of SARS-CoV PLpro, where it has been proven to play a crucial role in SARS-CoV PLpro inhibitor binding that inhibitor recognition specificity of MERS-CoV PLpro may differ from that of SARS-CoV PLpro. Inhibitory activity, of this compound was selective for SARS-CoV and MERS-CoV PLpro enzymes over two human homologues, and the ubiquitin C-terminal hydrolases [80]. The papain-like protease 1 ( $\left.\mathrm{PL1}^{\mathrm{pro}}\right)$ domain is present in nonstructual protein 3 (nsp3) of alpha corona viruses and subgroup $2 \mathrm{a}$ beta corona viruses, and the papain-like protease $2\left(\mathrm{PL} 2^{\mathrm{pro}}\right)$ is present in SARS-CoV. In combination with the prior characterization of PL2pro from other alpha corona-viruses of human corona viruses 229E, NL63, these viruses employ two $\mathrm{PL}^{\text {pro }} \mathrm{s}$ with overlapping specificities toward both viral and cellular substrates [81]. ZAPs also could probably inhibit the HCoVs that the ZAP could regulate RNA virus degradation of SARS-CoV's and MERS-CoV's RNA virus. $\mathrm{Zn}^{2+}$ ions are capable of inhibiting PLpro activity and the zinc conjugates to inhibit SARS-CoV PLpro activity that targeting PLpro with antiviral drug may have an advantage in not only inhibiting viral replication but also inhibiting the dys-regulation of signaling cascades infected cells, leading to cell death [82]. Further, zinc conjugated complexes as SARS-CoV 3C-like protease inhibitors play important role for this $\mathrm{Zn}^{2+}$ centered coordination pattern that the zinccoordinating inhibitor is tetrahedrally coordinated to the $\mathrm{His}^{40}-\mathrm{Cys}^{147}$ catalytic dyad of CVB3 3C ${ }^{\text {pro }}[83,84]$.

Most of the coronaviruses can cause the infectious diseases in human, currently, there are four coronaviruses: $\alpha-\mathrm{CoV}, \beta-\mathrm{CoV}, \gamma-\mathrm{CoV}$, and $\delta-\mathrm{CoV}$ that $\alpha-\mathrm{CoV}$ and $\beta-\mathrm{CoV}$ mainly infect the respiratory, gastrointestinal, and central nervous system of humans and mammals, while $\gamma-\mathrm{CoV}$ and $\delta$-CoV mainly infect the birds, in which 2019-nCoV or SARS-CoV-2 belongs to the $\beta-\mathrm{CoV}$ according to the phylogenic analysis based on the viral genome [85]. This $\beta-\mathrm{CoV}$ structure has a spike glycoprotein $(\mathrm{S})$ that the coronavirus protein mediates coronavirus entry into host cells which the evolution of these two critical function of coronavirus spike proteins, receptor recognition and membrane fusion must be considered [86]. It is responsible for binding 
to the receptor on the host cell as well as mediating the fusion of host and viral membrane, including with a process driven by major conformational changes of the $\mathrm{S}$ protein [87]. Zinc ion drug development is anticipated to be adopted by using ZAP Viral destruction via cell surface receptors [88] and $\mathrm{Zn}^{2+}$ coordination pattern [89]. Zinc ions may be functioned with the viral spike(S), envelope (E), necleocapsid $(\mathrm{N})$ proteins, and membrane $(\mathrm{M})$ in human coronavirus particles by $\mathrm{Zn}^{2+}$-centered tetrahedrally coordinated binding to these microproteins. Zinc is efficient for the prevention of respiratory ailment and pneumonia that the $\mathrm{N}$ protein of $\mathrm{HCoV}$ induces aggregation of human elongation factor 1-alpha, inhibiting protein translation and cytokinesis by blocking filamentous-actin bundling and proliferation of several steps in T-lymphocyte generation was significantly inhibited by the infection of recombinant retrovirus expressing $\mathrm{HCoV} \mathrm{N}$ protein, in which there will result in zinc ions-induced prevention of infectious and pneumonic spreading [90]. Accordingly, appreciable $\mathrm{Zn}^{2+}$ ion solutions may be most effective for the prevention of coronavirus infection, subsquent respiratory illness that the lower $\mathrm{Zn}^{2+}$ ion concentration $(<80 \mu \mathrm{M})$ could be efficient for vaccine candidates and the higher $\mathrm{Zn}^{2+}$ ion concentration $(\fallingdotseq 100 \mu \mathrm{M})$ should be available for the pulmonary disease and the infectious spreading.

As mentioned above, the virucidal activities of $\mathrm{ZNF}$, ZAP, ZBD, ZnO-NPs and prevention of coronavirus infection and respiratory ailment for virus entry, replication, and spread are represented in Table 2.

Table2. Virucidal activities of ZNF, ZAP, ZBD, and ZnONPs for prevention, virus entry, replication, and spread against $C M V, H C V, H E V, H I V, M L V$, and $H C o V s$

\begin{tabular}{|c|c|c|}
\hline $\mathrm{Zn}^{2+}$ ions & \multicolumn{2}{|c|}{$\begin{array}{l}\text { Virucidal activities of } \mathrm{Zn}^{2+} \text { from } \mathrm{ZNF} \text {, } \mathrm{ZAP}, \mathrm{ZBD} \text {, and } \mathrm{ZnONPs} \text { for } \\
\text { prevention, virus entry, replication, and spread against CMV, } \\
\text { HCV, HEV, HIV, MLV, and HCoVs }\end{array}$} \\
\hline \multirow[b]{2}{*}{$\mathbf{Z n}^{2+}$} & Adsorption/Entry & DNA/RNA Replication, Spread \\
\hline & $\begin{array}{l}\longrightarrow \mathrm{Zn}^{2+}, \cdot \mathrm{O}_{2}^{-}, \mathrm{H}_{2} \mathrm{O}_{2} \\
\cdot \mathrm{EBV}-i n d u c e d \text { zinc finger gene } \\
Z N F^{E B} \text { controls entry and exit } \\
\cdot \mathrm{ZBD} \text { prevent viral entry and } \\
\text { and } \mathrm{GPC} \text { inhibit activate } \\
\text { membrane fusion } \\
\cdot \mathrm{Zn} \text {-metalloprotease inhibits } \\
\text { entry and cell-cell fusion } \\
\cdot \mathrm{ZnOTs} \text { block HSV-2 } \\
\text { attachment } \\
\cdot \mathrm{ZnOTs} \text { inhibit HSV-1 entry } \\
\text { and spread } \\
\cdot 2,500 \mathrm{mg} / \mathrm{kg} \text { diet } \mathrm{ZnO} \text { has } \\
\text { antiviral activity of SARS-CoV } \\
\cdot \mathrm{ZnONPs} \mathrm{inhibit} \mathrm{H1N1} \\
\text { influenza virus entry } \\
\cdot \text { Lower } \mathrm{Zn}{ }^{2+} \text { conc may be } \\
\text { efficient for vaccine candidate } \\
\text { and higher } \mathrm{Zn}{ }^{2+} \text { conc may } \\
\text { prevent respiratory ailment } \\
\text { and acute pneumonia } \\
\text { spreading against } \mathrm{HCoVs}\end{array}$ & 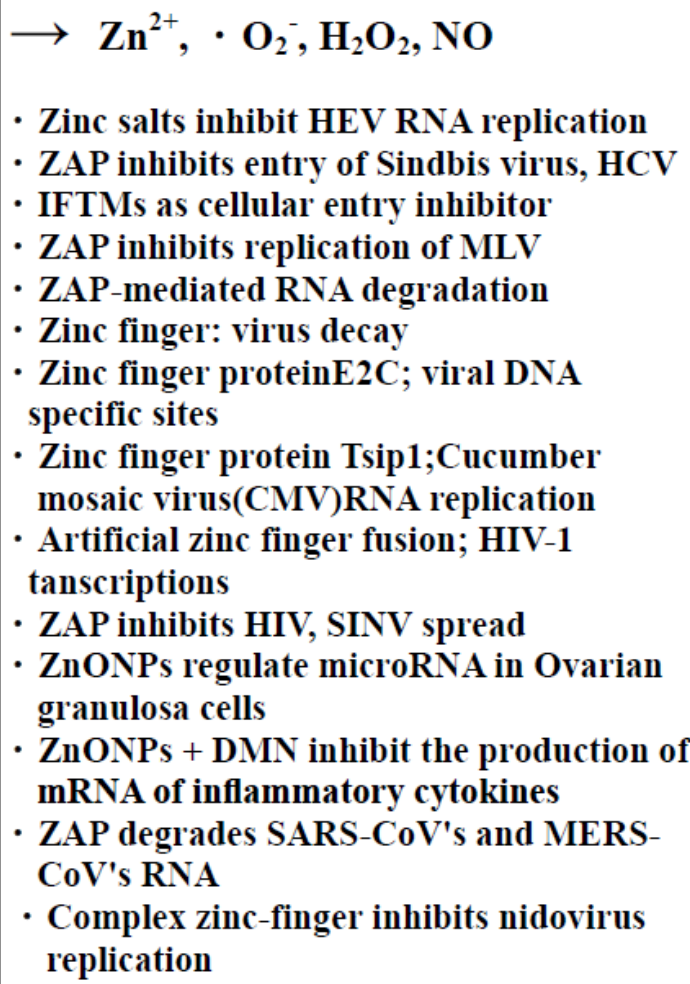 \\
\hline
\end{tabular}

\section{CONCLUSions}

Bacteriolyses by $\mathrm{Zn}^{2+}$ ions-induced activated PGN autolysins, virucides by ZAP. ZBD, and ZnONPs, and prevalent human coronaviruses have been discussed, and the bacteriolytic and virucidal mechanisms are clarified respectively.

Bacterial peptidoglycan (PGN) autolysin AmiA for S.aureus amidase is acted on PGN binding 
and cleavage. AmiA distinguishes PGN mostly by the peptide, and the cleavage is facilitated by a zinc-activated molecule. The autolytic activity of the recombinant amidase of the Aas (aut olysin/ adhesin of St aphylococcus saprophyticus) is inhibited and is neccesary for the C-terminal GW repeats.

Lytic amidase autolysin LytA associates with the cell wall via its zinc-binding motif. The LytB PGN hydrolase responsible for physical separation of daughter cells cleaves the GlcNAc- $\beta-(1,4)-M u r N A c$ glycosidic bond of PGN building units. LytC, LytD, and LytF are expressed in the same subpopulation of cells and complete flagellar synthesis.

Ami B catalyzes the degradation of PGN in Gram-negative bacteria, resulting in a marked increases of sensitivity to oxidative stress and organic acids. Amidase activity of amiC controls cell separation and PGN fragments release. Enterotoxigenic E.coli (ETEC) is the most common bacterial cause of children's diarrea, in which antigen and antitoxin antibodies that neutralized both toxins that are associated with all cases of ETEC diarrhea. Bacterial autolysins enable the bacteriolyses of bacterial cell walls trim cell surface PGN to prevent detection by bacterial innate immune system. Autolysin mediated bacteriolysis- and zinc dependent lysis-induced bacterial cell death can contribute to the bactericidal activities, where PGN autolysins interact with biomolecules causing cell apoptosis leading to cell death. Human peptidoglycan recognition proteins (PGRPs) are novel class of recognition and effector molecules with broad $\mathrm{Zn}^{2+}$ dependent bactericidal activity against both Gram-positive and Gram-negative bacteria. In these autolysins, amidases of zinc-dependent PGN autolysin are enhanced and induced the anti-bacterial activities against bact erial infection. Thus, autolysin-mediated bacteriolysis induced bacterial cell death can contribute to the bactericidal activities.

On the other hand, enveloped viruses enter cells and initiate disease-causing cycles of replication that in all cases virus-cell fusion is executed by one or more viral surface glycoproteins denoted as the fusion protein. The novel EBV-induced zinc finger gene $\left(Z N F^{E B}\right)$ controls entry and exit from cell cycling in activated lymphocytes. The designed polydactyl ZNF is prepared consiting HIV-1 type integrase fused to the synthetic zinc finger protein E2C. The zinc-finger antiviral protein (ZAP) specifically inhibits virus entry, replication, and spread of certain viruses, and ZAP inhibits the replication of certain viruses and promotes ZAP-mediated viral RNA degradation, The ZAP also inhibits Influenza A virus (IAV) protein expression. Furthermore, ZAP inhibits Retroviral RNA production and inhibits HIV-1 infection by promoting the degradation of specific viral mRNAs. ZAP specifically inhibits the replication of certain viruses and promotes viral RNA degradation.

ZAP' stress with antiviral activity and induced virus replication are regulated upon virus infection to inhibit virus spread. ZAP-70 kinase regulates HIV cell-to-cell spread that HIV usurps the immunological components to ensure its own spread through cell-to-cell contacts.

A novel zinc-binding domain (ZBD) is essential for formation of the functional Junin virus envelope glycoprotein complex.

Thus, ZNF, ZAP, and ZBD specifically inhibit virus entry, replication, and spread of many viruses. The host-virus interaction, conformational changes of specialized virus envelope proteins, and refolding reactions of specific fusion proteins in an essential steps entry, replication, and spread of enveloped virus life cycle have been worthy of remark in fascination that these diverse viral fusion ptotein could be used in next-generation for therapeutic intervention in arenaviral disease.

Zinc oxide and ZnONPs inhibit virus entry, replication, and spread. Zinc oxide tetrapods (ZnOTs) of micro-nanostructures block HSV-2 attachment, entry into host cell, and stop the spread of the virus among already infected cells. ZnONPs recently are used in various applications of veterinary science due to their antibacterial and antiviral agents, tissue repair that the ZnONPs are anticipated to be employed in prevention of human coronavirus infection. ZnONPs-5 $\mathrm{mg} / \mathrm{ml}$ specifically regulated the correlation of microRNAs and their targeted genes. ZnONPs to dimethylnitrosamine (DMN) treated rats inhibit the production of mRNA of inflammatory cytokines and reduce lipid peroxidation, oxidative stress and fibrosis in the liver.

Human coronaviuses (HCoVs) are recognised as coronaviruses (CoVs) associated with multiple respiratory diseases of varying severity, including common cold, pneumonia and bronchilitis that to date, seventh known $\mathrm{HCoVs}$ 
have been identified, namely HCoVs-229E, HCoV-NL63, HCoV-OC43, HCoV-HKU1, severe acute respiratory syndrome coronavirus (SARS-CoV), Middle East respiratory syndrome coronavirus (MERS-CoV), and recently newtyped 2019-nCoV, subsequent phylogenetic studies pointed to the bat origin of SARS-CoV based on sequences of SARS-like virus. New developed drugs for the 2019-nCoV has been now reported that Lopinavir/Ritonavir, Nucleoside analogues, Neuraminidase inhibitors, Remdesivir, peptide (EK1), abidol, RNA synthesis inhibitors (such as TDF, 3TC), anti-inflammatory drugs (such as hormones and other molecules), could be the drug treatment options for 2019-nCoV. However, the efficacy and safety of these drugs for 2019-nCoV still need to be further confirmed by clinical experiments. HCoVs are a well-known cause of respiratory infections that $\mathrm{Zn}^{2+}$ inhibits coronavirus and arterivirus RNA polymelase activity, and zinc ionophores block the virus replication that the combination of $\mathrm{Zn}^{2+}$ and pyrithione at low concentrations $\left(\mathrm{Zn}^{2+}\right.$ concentration $2 \mu \mathrm{M}$ ) inhibits the replication of SARS-CoV and arterivirus RNA.

Replication of SARS-CoV requires proteolytic processing of the replicase polyprotein by two viral cystein proteases, a chymotrypsin-like protease (3CLpro) and a papain-like protease (PLpro). The MERS-CoV PLpro blocking loop 2 (BL2) structure differs from that of SARSCoV PLpro, where it has been proven to play a crucial role in SARS-CoV PLpro inhibitor binding that inhibitor recognition specificity of MERS-CoV PLpro may differ from that of SARS-CoV PLpro. In addition, inhibitory activity, of this compound was selective for SARS-CoV and MERS-CoV PLpro enzymes over two human homologues, and the ubiquitin C-terminal hydrolases. ZAPs also could probably inhibit the HCoVs that the ZAP could regulate RNA virus degradation of SARS-CoV's and MERS-CoV's RNA virus. $\mathrm{Zn}^{2+}$ ions are capable of inhibiting PLpro activity and the zinc conjugates to inhibit SARS-CoV PLpro activity that targeting PLpro with antiviral drug may have an advantage in not only inhibiting viral replication but also inhibiting the dysregulation of signaling cascades infected cells, leading to cell death. Further, zinc conjugated complexes as SARS-CoV 3C-like protease inhibitors play important role for this $\mathrm{Zn}^{2+}$-centered coordination pattern that the zinc-coordinating inhibitor is tetrahedrally coordinated to the
His $^{40}-$ Cys $^{147}$ catalytic dyad of CVB3 $3 C^{\text {pro }}$. Most of the coronaviruses can cause the infectious diseases in human, currently, there are four coronaviruses: $\alpha-\mathrm{CoV}, \beta-\mathrm{CoV}, \gamma-\mathrm{CoV}$, and $\delta-\mathrm{CoV}$ that $\alpha-\mathrm{CoV}$ and $\beta-\mathrm{CoV}$ mainly infect the respiratory, gastrointestinal, and central nervous system of humans and mammals, while $\gamma-\mathrm{CoV}$ and $\delta$-CoV mainly infect the birds, in which 2019-nCoV or SARS-CoV-2 belongs to the $\beta-\mathrm{CoV}$ according to the phylogenic analysis based on the viral genome. This $\beta-\mathrm{CoV}$ structure has a spike glycoprotein (S) that the coronavirus protein mediates coronavirus entry into host cells which the evolution of these two critical function of coronavirus spike proteins, receptor recognition and membrane fusion must be considered. It is responsible for binding to the receptor on the host cell as well as mediating the fusion of host and viral membrane, including with a process driven by major conformational changes of the $\mathrm{S}$ protein. Zinc ion drug development is anticipated to be adopted by using ZAP Viral destruction via cell surface receptors and $\mathrm{Zn}^{2+}$ coordination pattern.

\section{REFERENCES}

[1] N. Z. Gammoh and L. Rink (2017); Zinc in infection and inflammation. Nutrients. 9: 1-25 pages.

[2] K-H. Ibs and L. Rink (2003); Immunity enhanced by trace elements. J. Nutr. 133: $1452 \mathrm{~S}-1456 \mathrm{~S}$.

[3] H. Steinbrenner, S. Al-Quraishy, M. A Dkhil et al (2015); Dietary selenium in adjuvant therapy of viral and bacterial infections. American Society for Nutrition, Adv. Nutr. 6: 73-82.

[4] Z. Varadyova, D. Mravcakova, M. Babjak et al, (2018); Effects of herbal nut raceut icsls and/or zinc against Haemonchus contourtus in lambs experimentally infected. BMC Veterinary Research. 14:1-12 pages.

[5] L. D. Palmer and E. Skaar (2016); Transition metals and virulence in bacteria, Аnnu Rev Genet, 50,No 23:67-94.

[6] L. M. Pium, L. Rink and Hajo Kaase (2010); The essential toxin: Impact of zinc on human health. International Journal of environmental research and public health. 7: 1342-1365.

[7] M. Lancellotti, R. F. C. Pereira, G. G. Cury and L. M. de Hollanda (2009); Pathogenic and opportunistic respiratory bacteria-induced apoptosis. The Brazilian Journal of Infectious Deseases, 13(3): 226-231.

[8] S. Dashti-Khavidaki, H. Khalili, S-M. Vahedi, M. Lessan-Pezeshki (2019); Serum zinc concentrations in patients on maintenance hemodialysis and its relationship with anemia, 
Bacteriolysis by Zinc Ions-Induced PGN Autolysins, Virucide by ZAP, ZBD, ZnONPs, and Prevaling Zinc Ions-Mediated HCoVs Matter

parathyroid hormone concentrations and pruritus severity. Saudi Journal of Kidney Diseases nd Transplantation. 21(4): 641-645.

[9] T. Ishida (2017); Antibacterial mechanism of bacteriolyses of bacterial infection cell walls by zinc ion induced activations of PGN autolysins, and DNA damages, Journal of Genes and Proteins, 1: 1-7.

[10] J. Humann and L. J. Humann and L. Lenz; Bacterial peptidoglycan degrading enzymes and their impact on host muropetide detection, $J$. Innate Immun, 2009; 1:88-97.

[11] M. L. Atilano, P. M. Pereira, F. Vaz et al (2014); Bacterial autolysins trim cell surface peptidoglycan to prevent detection by the Drosophila innate immune system. Elife Sciences. 2014; 3:1-23.

[12] Jose Maria Navarro and Mercedes Perez-Ruiz (2011); Antiviral immunity. Current Immuunology Review. 7: 19-24.

[13] C. A. McDevitt, A. D. Ogunnity, E. Valkov, et al (2011); A molecular mechanism for bacterial susceptibility to zinc. PLOS Pathogens. 2011; 7, Issue 11: 1-9.

[14] C-I Y. Ong, C. M. Gillen, T. C. Barnett, et al (2014); An antimicrobial role for zinc in innate immune defense against group A Streptococcus. The Journal of Infectious Diseases. 209: 15001508.

[15] J. C. King, FD. M. Shames and L. R. Woodhouse (2000); Zinc and health: Current status and future directions. Zinc homeostasis in humans. J. Nutr. 2000; 130: 1360S-1366S

[16] S. Overbeck, L. Rink, H. Haase (2008); Modulating the immune response by oral zinc supplementation: a single approach for multiple diseases. Arch. Immunol. Ther. Exp. 56: 15-30.

[17] J. M. Navarro and M. Perez-Ruiz (2011); Antiviral immunity. Current Immunology Reviews. 7: 19-24.

[18] S. A. Read, S. Obeid, C. Ahlendtiel,and G. Ahlenstiel (2019); The role of zinc in antiviral immunity. Adv Nutr, 0:1-15.

[19] Felix A. Rey and Shee-Mei Lok (2018); Common features of enveloped viruses and implications for immunogen design for next-generation vaccines. Cell. 172, Issue 6: 1319-1334.

[20] T. Ishida (2016); Bacteriolyses of $\mathrm{Cu}^{2+}$ solution on bacterial cell walls/cell membrane and DNA base pairing damages, Japanese Biomedical Research on Trace Elements, 27, No.4:151-161.

[21] T. Ishida (2019); Comparative bacteriolytic mechanism for $\mathrm{Ag}^{+}$and $\mathrm{Zn}^{2+}$ ions against S.aureus and E. coli: A review. Annals of Microbiology and Infectious Diseases. 2, Issue 1: 1-12.

[22] A. Vermassen, S. Leroy, Regine Talon et al (2019); Cell wall hydrolases in bacteria: Insight on the diversity of cell wall amidases, glycosidases and peptidases toward peptidoglycan. Frontiers in Microbiology. 10, article 331:1-27.

[23] F. M. Buttner, S, Zoll, M. Nega, et al (2014); Structure-function analysis of S.aureus amidase reveals the determinant of peptidoglycan recognition and cleavage. Journal of Biological Chemistry. 289(16): 11083-11095.

[24] W. Hell, S. Reichl, A. Agnes, S. Gatermann (2003); The autolytic activity of the recombinant amidase of St aphylococcus saprophyticus is inhibited by its own recombinant GW repeats. FEMS Microbiology Letters. 227: 47-51.

[25] P. Mellroth, T. Sandalova, A. Kikhney et al (2014); Structural and functional insights into peptidoglycan access for the Lytic amidase LytA of Streptococcus pneumoniae. Mbio. Asm. Org. 5, Issue 1: 1-10.

[26] C. C. Kietzman, G. Gao, B. Mann et al (2016); Dynamic capsule restructuring by the main pneumococcal autolysin LytA in response to the epithelium. Nature Communications. 108:1-9.

[27] P. Rico-Lastres, R. Diez-Martinez, M. IglesiasBexiga, et al (2015); Substrate recognition and catalysis by LytB, a pneumococcal peptidoglycan hydrolase involved in virulence. Scientific Reports. 5: 1- 17.

[28] R. Chen, S. B. Guttenplan, K. M. Blair, and D.B. Kearns (2009); Role of the $\sigma^{\mathrm{D}}$-dependent autolysins in Bacillus subtilis population heterogeneity. Journal of Bacteriology. 191, No.18: 5775-5784.

[29] S.Zoll, M. Schlag, A. V. Shkumatov, M. Rautenberg, et al (2012); Ligand-binding properties and conformational dynamics of autolysin repeat domains in Staphylococcal cell wall recognition. Journal of Bacteriology. 194, No.11: 3789-3802.

[30] [30] Vineet K. Singh (2014); High levelexpression and purification of Atl, the major autolytic protein of Staphylococcus aureus. International Journal of Microbiology. 2014(4): 1-7.

[31] E. K. Stinemetz, P. Gao, K. L. Pinkston et al (2017); Processing of the major autolysin of $E$. faecalis, AtlA, by the zinc metallo-protease, GelE, impacts AtlA septal localization and cell separation. PLOS ONE. 12(10) :1-17.

[32] P. Houston, S.E. Rowe, C. Pozzi, et al (2011); Essential Role for the major autolysin in the fibronectin-binding protein-mediated Staphylococcus aureus biofilm phenotype. Infection and Immunity. 70, No.5:1153-1165.

[33] V. Dubee, F. Chau, M. Arthur, et al (2011); The in vitro contribution of autolysins to bacterial killing elicited by amoxicillin increases with inoculum size in Entetrococcus faecalis. 
Antimicrobial Agents and Chemotherapy. 55, No.2: 910-912.

[34] R. Ohnishi, S. Ishikawa,and J. Sekiguchi (1999); Peptidoglycan hydrolase LytF plays a role in cell separation with CwIF during vegetative growth of Bacillus subtilis. Journal of Bacteriology. 181, No. 10: 3178-3184.

[35] A. Delaune, O. Poupel, A. Mallet, et al (2011); Peptidoglycan crosslinking relaxation plays an important role in Staphylococcus aureus walkrdependent cell viability. PLOS ONE. 6, Issue2: $1-10$.

[36] D. C. Osipovitch and K. E. Griswold (2015); Fusion with a cell wall binding domain renders autolysin LytM a potent anti-Staphylococcus aureus agent. FEMS Microbiology Letter. 362: 1-7.

[37] S. G. Murphy, L. Alvarez, M. C. Adams et al (2019); Endopeptidase regulation as a novel function of the Zur-dependent zinc starvation response. MBio. 10, issue 1: 1-15.

[38] Ahn, Sun-Hee, D-G Kim, S-H. Jeong et al (2006); Isolation of N-acetylmuramoyl-Lalanine amidase gene( $\mathrm{amiB})$ from Vibrio anguillarum and the effect of amiB gene deletion on stress responses. J. Microbiol. Biotechnol. 2006; 16(9): 1416-1421.

[39] J. D. Lenz, E. A. Stohl, R. M. Robertson, K. T. Hackett et al; Amidase activity of AmiC controls cell separation and stem peptide release and is enhanced by NlpD in Neisseria genorrhoeae. Journal of Biological Chemistry. 291, No.20: 10916-10933.

[40] Z. TR. Lonergan, B. L. Naim, J. Wang et al (2019); An acinetobacter baumanni, zincregulated peptidase maintains cell wall integrity during immune-mediated nutrient sequestration. Cell Reports. 26:2009-2018.

[41] A. Raz, N. D. Djadid, S. Zakeri (2013); Molecular characterization of the carboxypeptidase B1 of Anopheles stephensi and its evaluation as a target for transmissionblocking vaccines. Infection and Immunity. 81, No.6: 2206-2216.

[42] A. P. Frasch, A. K. Carmona, L. Juliano, et al (2012); Characterization of the M32 met allocarboxy-pept idase of Trypanosoma brucei: differences and similarities with its orthologue in Trypanosoma cruzi. Mol Biochem Parasitol. 184(2): 63-70.

[43] M. Wang, L-H. L. Liu, S. Wang, et al (2007); Human peptidoglycan recognition proteins require zinc to kill both Gram-positive and Gram-negative bacteria and are synergistic with antibacterial peptides, The Journal of Immunology. 178: 3116-3125.

[44] N. Kaushik, C. Subramani, S. Anang, et al (2017); Zinc salts block hepatitis E virus replication by inhibiting the activity of viral RNA-dependent RNA polymerase. Journal of Viology. 91, Issue 21: 1-13.

[45] R.O. Suara and J. E. Crowe, Jr. (2004); Effectof zinc salts on respiratory syncytial virus (RSV) replication. Antimicrobial Agents and Chemotherapy. 48(3): 783-790.

[46] C. E. Tune, M. Pilon, Y. Saiki, and H-M. Dosch (2002); Sustained expression of the novel EBVinduced zinc finger gene, $\mathrm{ZNF}^{\mathrm{EB}}$, is critical for the transition of $\mathrm{B}$ lymphocyte activation to oncogenic growth transformation.The Journal of immunology. 168: 680-688.

[47] W. Tan, K. Zhu, D.J. Segal, et al (2004); Fusion protein consisting of HIV type 1 integrase and the designed polydactyl zinc finger protein E2C firect integration of viral DNA into specjfic sites. Journal of Virology. 78, No.3: 1301-1313.

[48] H. Lian, R. Zang, J. Wei, et al (2018); The zinc-finger protein ZCCHC3 binds RNA and facilitates viral RNA sensing and activation of the RIG-I-like receptors. Immunity. 49: 438-448.

[49] Y.-S. Kim, J.-M. Kim, D,-L. Jung, et al (2005); Artificial zinc finger fusions targeting Sp1binding sites and the trans-activator-responsive element potently repress transcription and replication of HIV-1. The Journal of Biological Chemistry. 280,No.22: 21545-21552.

[50] S. U. Hur, M. J. Kim, B. Kook, et al (2011); A zinc finger protein Tsip1 controls Cucumber mosaic virus infection by interacting with the replication complex on vacuolar membranes of the tobacco plant. New Phytologist. 191: 746-762.

[51] THE ROCKEFELLER UNIVERSITY; Virus entry and virus-host interactions. Laboratory of Virology and Infectious Disease. 2019; December 15: 1 page.

[52] W-Y. J. Lee, R. Menhua, C. Liang \& R. D. Sloan (2018); IFITM proteins inhibit HIV-1 protein synthesis. Scientific Reports. 8:1-15 pages.

[53] K. M. Crosse, E. Monson, M.R. Beard, K. J. Helbig (2018); Interferon-simulated genes as enhancers of antiviral innate immune signaling. Journal of Innate Immunity. 10: 85-93.

[54] M. J. Bick, J-W. N. Carroll, et al, (2003); Expression the zinc-finger antiviral protein inhibits Alphavirus replication. Journal of Virology. 77, No.21: 11555-11562.

[55] Q. Tang, X. Wang, G. Gao (2017); The short form of the zinc finger antiviral protein inhibits influenza A virus porotein expression and is antagonized by the virus-encoded NS1. Journal of Virology. 91, Issue 2: 1-14 pages

[56] F. Villalon-Letelier, A. G. Brooks, P. M. Saunders, et al (2017); Host cell restriction factors that limit influenza A infection. Viruses. 9(12):1-18pages. 
[57] J. M. Schapiro, S. J. Libby, and F. Fang (2003); Inhibition of bacterial DNA replication by zinc mobilization during nitrosative stress. PNAS. 2003; 100, No.14: 8496-8501.

[58] X. Wang, F. Lv and G. Gao (2010); Mutagenesis analysis of the zinc-finger antiviral protein. Retrovirology. 7: 1-9 pages.

[59] G. Gao, X. Guo, S. P. Goff (2002); Inhibition of Retroviral RNA production by ZAP, a CCCH-type zinc finger protein. SCIENCE, 297: 1703-1706.

[60] V. Zhu, G. Chen, F. Lv, X. Wang, et al (2011); Zinc-finger antiviral protein inhibits HIV-1 infection by selectively targeting multiply spliced viral mRNA for degradation. PNAS. 2011; 108, No.38: 215834-15839.

[61] L. M. John Law, B. S. Razooky, M. M. H. Li, et al (2019); ZAP's stress granule localization is correlated with its antiviral activity and induced by virus replication. PLOS Pathogens.15(5): 1-22.

[62] Nathalie Sol-Foulon, Marion Sourisseau1, Franc, oise Porrot1, Maria-Isabel Thoulouze, et al (2007); ZAP-70 kinase regulate HIV cell-tocell spread and virological synapse formation. The EMBO Journal. 26: 516-526.

[63] W. Mothes, N. M. Sherer, J. Jin, P. Zhong (2010); Virus cell-to-cell transmission. Journal of Virology. 84, No.17: 8360-8368.

[64] Joanne York and Jack H. Nunberg (2007); A novel zinc-binding domain is essential for formation of the functional Junin-virus envelope glycoprotein complex. Journal of Virology. 81, No.24: 13385-13391.

[65] K. Briknarova, C. J. Thomas, J. York, and H. Nunberg (2012); Structure of a zinc-binding Domain in the Junin virus envelope glycoprotein. The Journal of Biological Chemistry. 286, No.2: 1528-1536.

[66] A. Seybert, C. C. Posthuma, L. C. van Dinten, E. J. Snijder (2006); A complex zinc finger controls the enzymatic activities of Nidovirus helicases. Journal of Virology. 79(2): 696-704.

[67] J. M. White, S. E. Deolos, M. Brecher, and K. Schornberg (2008); Structure and mechanisms of viral membrane fusion proteins: Multiple variations on a common theme. Crit Rev Biochem Mol Biol. 44(3): 189-219.

[68] T. Antoine, Y. K. Mishra, J. Trigilic, et al (2012); Prophylactic, therapeutic and neutralizing effects of zinc oxide tetrapod structures against herpes simplex virus type-2 infection. Antiviral Res. 96(3): 363-375.

[69] N. Duggal, D. Jaishankar, T. Yadavalli, et al (2017); Zinc oxide tetrapods inhibit herpes simplex virus infection of cultured corneas. Molecular Vision. 23; 26-38.

[70] W. Chai, S. S Zakrzewski, D. Gunzel, et al (2014); High-dose dietary zinc oxide mitigates infection with transmissible gastroenteritis virus in piglets. BMC Veterinary Research. 10: 1-10.

[71] H. Ghaffan, A. Tavakoli, A. Moradi, et al (2019); Inhibition of H1N1 influenza virus infection by zinc oxide nanoparticles: another emerging application of nanomedicine. Journal of Biomedical Science. 26: 1-10.

[72] Y. Zhao, L. Li, L-J. Min, et al (2016); Regulation of microRNAs, and the correlations of microRNAs and their targeted genes by zinc oxide nanoparticles in ovarian granulosa cells. PLOS ONE. 10: 1-18.

[73] V. Rani, Y. Verma, K. Rana, et al (2018); Zinc oxide nanoparticles inhibit dimethylnitrosamine induced liver injury in rat. Chemico-Bological Interactions. 295: 84-92.

[74] C.C. Posthuma, A. J. W. te Velthuis, E. L. Snijder (2017); Nidovirus RNA polymerases: Complex enzymes handling except ional RNA genomes. Virus Research. 234:58-73.

[75] Y. X.Lim, Y. L.Ng, J. P. Tam and D.X. Liu (2016); Human coronaviruses:A review of virus-host interactions. Diseases. 4: 1-28pages.

[76] Hongzhou Lu (2020); Drug treatment options for the 2019-new coronavirus (2019-nCoV). BioScience Trends Advance Publication : DOI 10.5582/bst. 01020: P1-P3.

[77] A. J. W. te Velthuis, S. H. E. van den Worm, A. C. Sims, et al (2010); $\mathrm{Zn}^{2+}$ inhibits coronavirus and arterivirus RNA polymerase activity in vitro and zinc ionophores block the replication of these viruses in cell culture. PLOS Pathogen. 6, Issue 11: 1-10.

[78] S. L. Warnes, Z. R. Littie, C. W. Keevil (2015); Human coronavirus 229E remain infectious on common touch surface material. Mbio 6,Issue 6: 1-10.

[79] K. Ratia, K. Singh Saikatendu, B. D. Santarsiero, et al (2006); Severe acute respiratory syndrome coronavirus papain-like protease: Structure of a viral deubiquitinating enzyme. PNAS. 103, No.25: 58717-5722.

[80] H. Lee, H. Lei, B. D. Santarsiero, J. L. Gatuz, et al (2015); Inhibitor recognition specificity of MERS-CoV papain-like protease differs from that of SARS-CoV. ACS Chem Biol. 10(6): 1456-1465.

[81] J. A. Wojdyla, I. Manolaridis, P. B. van Kasteren, et al, (2020); Papain-like protease 1 from transmissible gastroenteritis virus: Crystal structure and enzymatic activity toward viral and cellular substrates. Journal of Virology. 84, No.19: 10063-10073.

[82] Y. M. Baez-Santos, S. E. John and A. D. Mesecar (2015); The SARS-Coronavirus papain-like protease: Structure, function and inhibition by designed antiviral compounds. Antiviral Res. 115: 21-38. 
Bacteriolysis by Zinc Ions-Induced PGN Autolysins, Virucide by ZAP, ZBD, ZnONPs, and Prevaling Zinc Ions-Mediated HCoVs Matter

[83] C-C. Lee, C-J. Kuo, M-F. Hsu, et al (2007); Structural basis of mercury- and zincconjugated complexes as SARS-CoV 3C-like protease inhibitors. FEBS Letters. 581: 54545458.

[84] C-C. Lee, C-J. Kuo, T-P. Ko, M-F. Hsu, et al (2009); Structural basis of inhibition specificities of 3C and 3C-like proteases by zinc-coordinating and peptidomimetic compounds. Journal of Biological Chemistry. 284(12): 7646-7655.

[85] X. Peng, X. Xu, Y. Li, et al (2020); Transmission routes of 2019-nCoV and controls in dental practice. International Journal of Oral Science. 12(9): 1-6.

[86] Fang Li (2016); Structure, function, and evolution of coronavirus spike proteins. Annu Rev Virol. 3(1): 237-261.

[87] S.Belouzard, J. K. Miller, B. N. Licitra and G. R. Whittaker (2012); Mechanism of corona virus cell entry mediated by the viral spike protein. Viruses. 4(6): 1011-1033.

[88] T. Ishida (2020); Zinc Immune Anti-Infectious Activities of Bacteriolysis by $\mathrm{Zn}^{2+}$-induced Bacterial PGN Autolysins and ZAP's Viral Destruction by Cell Surface Receptors. Biomedical Research and Reviews. 3(2):1-8.

[89] T. ISHIDA (2020); Virucidal Activities of Zinc-Finger Antiviral Proteins and ZincBinding Domains for Virus Entry, DNA/RNA Replication and Spread. Edelweiss Journal of Biomedical Research and Review. Volume 2 Issue 1 | PDF 109 | Pages 1-5.

[90] B. Zhou, J. Liu, Q. Wang, X. Liu, et al (2008); The nucleocapsid protein of SARS coronavirus inhibit cell cytokinesis and proliferation by intracting with translation elongation factot $1 \alpha$. Journal of Virology. 83: No.14: 6962-6971.

Citation: Dr. Sci. Tsuneo Ishida, "Bacteriolysis by Zinc Ions-Induced PGN Autolysins, Virucide by ZAP, $Z B D, Z n O N P s$, and Prevaling Zinc Ions-Mediated HCoVs Matter”, International Journal of Research Studies in Medical and Health Sciences. 2020; 5(3): 01-15.

Copyright: (C) 2020 Dr. Sci. Tsuneo Ishida, This is an open-access article distributed under the terms of the Creative Commons Attribution License, which permits unrestricted use, distribution, and reproduction in any medium, provided the original author and source are credited. 